\title{
ON A CONJECTURE FOR OSCILLATION OF SECOND ORDER ORDINARY DIFFERENTIAL SYSTEMS
}

\author{
ANGELO B. MINGARELLI ${ }^{1}$
}

\begin{abstract}
We present here some results pertaining to the oscillatory behavior at infinity of the vector differential equation
\end{abstract}

$$
y^{\prime \prime}+Q(t) y=0, \quad t \in[0, \infty),
$$

where $Q(t)$ is a real continuous $n \times n$ symmetric matrix function.

It has been conjectured (cf., e.g. [б]) that the criterion

$$
\lim _{t \rightarrow \infty} \lambda_{1}\left\{\int_{0}^{t} Q(s) d s\right\}=\infty
$$

where $\lambda_{1}(\cdot)$ denotes the maximum eigenvalue of the matrix concerned, implies oscillation. We show that this is so under the tacit assumption

$$
\liminf _{t \rightarrow \infty} t^{-1} \operatorname{tr}\left\{\int_{0}^{t} Q(s) d s\right\}>-\infty
$$

where $\operatorname{tr}(\cdot)$ represents the trace of the matrix under consideration.

1. We will be mainly concerned with the differential equation

$$
y^{\prime \prime}+Q(t) y=0, \quad t \in J,
$$

for a vector $y$ where $Q(t)=Q^{*}(t)$ is a real $n \times n$ symmetric matrix continuous on some interval $J$. Points $\alpha \neq \beta$ in $J$ will be called (mutually) conjugate relative to (1.1) provided there exists a solution $y(t) \neq 0$ of (1.1) which vanishes at $\alpha$ and $\beta$. The equation (1.1) will be called disconjugate on $[a, b]$ if there are no conjugate points therein, i.e., if every nontrivial solution vanishes at most once in $[a, b]$. When $J=[0, \infty)$ it will be termed oscillatory at $\infty$ if for every $a>0$ there exists $b>a$ such that (1.1) fails to be disconjugate on $[a, b]$. It will be nonoscillatory otherwise.

Associated with (1.1) is the matrix differential system

$$
Y^{\prime \prime}+Q(t) Y=0, \quad t \in J,
$$

for a matrix $Y$ where $Q$ is as in (1.1). A solution $Y(t)$ of (1.2) is said to be nontrivial if det $Y(t) \neq 0$ for at least one $t \in J$. A simple differentiation shows that whenever $Y$ is a solution of (1.2)

$$
Y^{*}(t) Y^{\prime}(t)-Y^{* \prime}(t) Y(t)=K
$$

for $t \in J$ where $K$ is a constant matrix. A nontrivial solution $Y$ will be called prepared or self-conjugate (cf. [4]) if $K=0$ in (1.3), i.e., $Y^{*} Y^{\prime}=\left(Y^{*} Y^{\prime}\right)^{*}$.

Received by the editors August 5, 1980.

1980 Mathematics Subject Classification. Primary 34A30, $34 \mathrm{Cl0}$.

Key words and phrases. Conjugate points, disconjugacy, oscillation at infinity, differential systems.

${ }^{1}$ Dedicated to my brother Marco Mingarelli. 
We now follow [5] in defining the space $S$ as the linear space of all real symmetric $n \times n$ matrices. Whenever $A \in S$, its eigenvalues (all necessarily real) will be denoted by $\lambda_{k}(A), 1<k<n$, where we take it that $\lambda_{1}(A)$ (resp. $\lambda_{n}(A)$ ) indicates the largest (resp. smallest) eigenvalues of $A$. The trace of $A$ will be designated by $\operatorname{tr} A$.

The following lemma incorporates results from matrix theory which we include for reference.

LEMMA 1.1. Let $A, B \in S$. Then both $\lambda_{1}, \lambda_{n}: S \rightarrow \mathbf{R}$ are nonlinear functionals with the following properties:

(i) $\lambda_{1}(A+B) \leqslant \lambda_{1}(A)+\lambda_{1}(B)$ and $\lambda_{1}(\mu A)<\mu \lambda_{1}(A)$ whenever $\mu>0$ is real.

(ii) $\lambda_{n}(A+B) \geqslant \lambda_{n}(A)+\lambda_{n}(B)$ and $\lambda_{n}(\mu A) \geqslant \mu \lambda_{n}(A)$ for $\mu>0$.

(iii) Whenever $A$ is integrable,

$\lambda_{1}\left[\int_{a}^{b} A(s) d s\right] \leqslant \int_{a}^{b} \lambda_{1}[A(s)] d s ; \quad \lambda_{n}\left[\int_{a}^{b} A(s) d s\right]>\int_{a}^{b} \lambda_{n}[A(s)] d s$.

(iv) If $A>B$ (i.e., $A-B$ is positive definite) we have $\lambda_{k}(A)>\lambda_{k}(B)$ for each $k$, $1<k \leqslant n$.

Moreover the trace $\operatorname{tr}: S \rightarrow \mathbf{R}$ is a positive linear functional on $S$ (cf. [3]) and therefore the Schwarz-type inequality holds:

for $A \in S$.

$$
|\operatorname{tr} A|^{2}<n \operatorname{tr}\left(A^{2}\right)
$$

Proof of Lemma 1.1. (i) and (ii) follow from the min-max characterizations of $\lambda_{1}$ and $\lambda_{n}$ and an application of the subadditivity and superadditivity of the "sup" and "inf" operations for real-valued functions (cf. e.g. Chapter 7 of [1]). Part (iii) essentially follows from (i) and (ii), (see also [8, p. 522, exercise 3]). Now the min-max representation of $\lambda_{k}, 1 \leqslant k \leqslant n$, also allows an easy proof of (iv) once the following inequality is used:

$$
\lambda_{k}(A+B) \geqslant \lambda_{k}(A)+\lambda_{n}(B)
$$

valid for all $A, B \in S, B \geqslant 0$, and $k=1,2, \ldots, n$ (cf. [1, p. 117, Theorem 3 and exercise]). Finally we note that (i) and (ii) both imply that $\lambda_{1}, \lambda_{n}$ are continuous functionals on $S$.

Let $A(t) \in S$ be a matrix function defined on $[0, \infty)$ and suppose that

$$
A\left(t_{2}\right)>A\left(t_{1}\right), \quad T<t_{1}<t_{2}<\infty .
$$

Then $\lambda_{k}\left[A\left(t_{2}\right)\right]>\lambda_{k}\left[A\left(t_{1}\right)\right], 1 \leqslant k \leqslant n$, and so the real valued function $\lambda_{k}[A(t)]$ is increasing for large $t$. We therefore obtain

Corollary 1.1. Let $A(t) \in S$ satisfy (1.5). Then

$$
\lim _{t \rightarrow \infty} \lambda_{k}[A(t)]
$$

exists for each $k, k=1,2, \ldots, n$, (may be infinite).

We also note that when $A=A^{*}$,

$$
\lambda_{1}\left(A^{*} A\right)>\left|\lambda_{1}(A)\right|^{2}
$$

as can be readily verified. 
Finally if $A \in S$ is nonnegative definite, $A>0$, the following bounds hold:

$$
\lambda_{1}(A) \leqslant \operatorname{tr} A<n \lambda_{1}(A),
$$

and in fact for any matrix $A \in S$ one always has

$$
\lambda_{n}(A)<\operatorname{tr} A / n<\lambda_{1}(A) \text {. }
$$

2. We now derive a matrix analog of a corresponding result for a scalar differential equation (cf. e.g. [2, p. 15, Lemma 3]).

LemMA 2.1. Let $V(t)=V^{*}(t)$ be a continuous $n \times n$ matrix function on $\left[t_{0}, \infty\right)$ and suppose that

$$
\operatorname{tr}\left\{V(t)+\int_{t_{0}}^{t} V^{2}(s) d s\right\}<M t
$$

for all $t \geq t_{1}$, where $M$ is a fixed constant. Then

$$
\liminf _{t \rightarrow \infty} t^{-1} \lambda_{1}\left\{\int_{t_{0}}^{t} V^{2}(s) d s\right\}<\infty
$$

REMARK 1. Whenever the limit as $t \rightarrow \infty$ of the left side of (2.1) exists (and is finite) it follows that the ensuing limit exists and

$$
\lim _{t \rightarrow \infty} \operatorname{tr}[V(t)]=0
$$

because of (1.4). Thus if $V(t)>0$ for large $t,(2.3)$ and (1.8) together will imply that

$$
\lim _{t \rightarrow \infty} V(t)=0 \text {. }
$$

REMARK 2. We note that since $V^{2}(t)>0$, so is its integral and therefore, by (1.8), (2.2) is equivalent to

$$
\liminf _{t \rightarrow \infty} t^{-1}\left\|\int_{t_{0}}^{t} V^{2}(s) d s\right\|<\infty .
$$

Moreover (2.1) cannot, in general, be weakened to

$$
\lambda_{n}\left\{V(t)+\int_{t_{0}}^{t} V^{2}(s) d s\right\}<M t
$$

for $t \geqslant t_{1}$ without affecting the conclusion. For example let $V(t)=\operatorname{diag}\{t, 0\}$. Then a simple calculation shows that the left side of $(2.4)$ is identically zero. However (2.2) fails in this case.

Nor will it be possible to expect that (2.2) holds if, say,

$$
\limsup _{t \rightarrow \infty} \operatorname{tr}\left\{V(t)+\int_{t_{0}}^{t} V^{2}(s) d s\right\}=\infty .
$$

To see this, let $V(t)=V^{*}(t)$ be the matrix $\operatorname{diag}\{t,-t\}$. In this case (2.2) fails on account of (1.8).

It is known (cf., e.g. [3]) that (1.1) is oscillatory at $\infty$ if

$$
\lim _{t \rightarrow \infty} \operatorname{tr}\left\{\int_{t_{0}}^{t} Q(s) d s\right\}=\infty
$$


We compare this with the scalar case of (1.1) and note that

$$
\liminf _{t \rightarrow \infty} t^{-1} \operatorname{tr}\left\{\int_{t_{0}}^{t} Q(s) d s\right\}=-\infty
$$

may or may not, by itself, imply oscillation. For example setting $Q(t)=$ $\operatorname{diag}\{t,-2 t\}$ we find that $(2.6)$ is satisfied and, in fact, (1.1) is oscillatory, as a simple calculation will show. On the other hand if we set $Q(t)=\operatorname{diag}\{-t,-2 t\}$, (2.6) will be satisfied once more but (1.1) will now be nonoscillatory and, in fact, disconjugate on $[0, \infty)$.

It was conjectured, cf. [6], that (1.1) is oscillatory at $\infty$ whenever

$$
\lim _{t \rightarrow \infty} \lambda_{1}\left\{\int_{0}^{t} Q(s) d s\right\}=\infty \text {. }
$$

This conjecture has been completely verified in the special cases (a), when $Q(t)>0$ and (b), when $Q(t) \equiv Q$ is a constant matrix (cf. [6]). In the following pages we settle this conjecture on the assumption that (2.6) is not satisfied, i.e.,

$$
-\infty<\liminf _{t \rightarrow \infty} t^{-1} \operatorname{tr}\left\{\int_{0}^{t} Q(s) d s\right\}
$$

TheOREM 2.1. Let $Q(t) \in S$ be continuous on $[0, \infty)$ and assume that (2.8) holds. Then (2.7) implies (1.1) is oscillatory at $\infty$.

REMARK 3. The condition

$$
\liminf _{t \rightarrow \infty} \lambda_{n}\left\{\int_{0}^{t} Q(s) d s\right\}>-\infty
$$

in conjunction with (2.7) easily implies that (1.1) is oscillatory. For

$$
\operatorname{tr}\left\{\int_{0}^{t} Q(s) d s\right\}=\lambda_{1}\left\{\int_{0}^{t} Q(s) d s\right\}+\Lambda(t)
$$

where $\Lambda(t)$, the sum of the remaining eigenvalues, is bounded below because of (2.9). Consequently (2.7) and (2.9) together imply (2.5). Hence (1.1) is oscillatory.

The conjecture can be essentially subdivided into two principal cases.

Case 1 (2). To verify the validity of the conjecture under the assumption (2.7) and (2.8) (resp. (2.6) and (2.7)).

Case 1 is completely solved in Theorem 2.1 above, while the answer to Case 2, in general, remains unknown at this point. (The case $Q(t) \equiv Q$ a constant matrix, comes under the latter heading if $\operatorname{tr} Q<0$. For such matrices Case 2 is verified as we have seen earlier.)

It appears highly probable that there is a positive answer to Case 2 in general, however the justification for this appears to be beyond the scope of the methods presented herein.

3. Proof of Lemma 2.1. We note that since $V=V^{*}, V^{2}(t)>0$, hence

$$
\int_{t_{0}}^{t} V^{2}(s) d s>0, \quad t>t_{0}
$$

If we now write $A(t)$ for the matrix in (3.1) we see that (1.5) holds, hence Corollary 1.1 implies that $A(t)$ converges to a possibly infinite limit matrix as $t \rightarrow \infty$. 
Furthermore a combination of (1.8) and (3.1) shows that (2.2) will be satisfied if and only if

$$
\liminf _{t \rightarrow \infty} t^{-1} \operatorname{tr}\left\{\int_{t_{0}}^{t} V^{2}(s) d s\right\}<\infty
$$

We therefore suppose, on the contrary, that (3.2) fails so that with $M$ as in (2.1) and

$$
W(t) \equiv \operatorname{tr}\left\{\int_{t_{0}}^{t} V^{2}(s) d s\right\}-M t
$$

we can choose $t$ so large that

$$
W(t)>t
$$

Now (2.1) implies that

$$
t M>\operatorname{tr}[V(t)]+\operatorname{tr}\left\{\int_{t_{0}}^{t} V^{2}(s) d s\right\}, \quad t>t_{1},
$$

and so (3.3) gives

$$
W(t)<-\operatorname{tr}[V(t)], \quad t>t_{1} .
$$

Hence for $t>t_{2}$, we must have

$$
\begin{aligned}
t^{2} & \leqslant W(t)^{2} \leqslant \operatorname{tr}[V(t)]^{2} \\
& \leqslant n \operatorname{tr}\left[V^{2}(t)\right]=n\left[W^{\prime}(t)+M\right] .
\end{aligned}
$$

Separating the variable in (3.5) and integrating over $\left[t_{2}, t\right]$ we find, using the estimate $W(t)>t$ in the integral,

$$
M\left(\frac{1}{t_{2}}-\frac{1}{t_{1}}\right)-\frac{1}{W(t)}+\frac{1}{W\left(t_{2}\right)}>\frac{1}{n}\left(t-t_{2}\right), \quad t>t_{2},
$$

but since $W(t)>0$ for such $t$, (3.6) gives a contradiction for large $t$. It now follows that (3.2) and hence (2.2) hold.

Proof OF TheOrem 2.1. We assume, on the contrary, that (1.1) is not oscillatory at $\infty$. Then there exists an $a>0$ such that (1.1) is disconjugate on $[a, b]$ for every $b>a$, i.e., $(1.1)$ is disconjugate on $[a, \infty)$. Hence, by $[4$, p. 388, Theorem 10.2] there exists a nontrivial prepared solution $Y(t)$ of $(1.2)$ such that det $Y(t) \neq 0$ on $(a, \infty)$. Defining

$$
V(t)=Y^{\prime}(t) Y^{-1}(t), \quad t>a,
$$

we see that $V(t)=V^{*}(t)$, since $Y$ is prepared, and a simple calculation shows that $V(t)$ satisfies the matrix Riccati equation

$$
-V^{\prime}(t)=Q(t)+V^{2}(t), \quad t>a .
$$

Integration of (3.7) over [ $a, t]$ now gives

$$
-V(t)+V(a)=\int_{a}^{t} Q(s) d s+\int_{a}^{t} V^{2}(s) d s .
$$

Since the last integral on the right of (3.8) is positive indefinite we have

$$
-V(t)+V(a) \geq \int_{a}^{t} Q(s) d s, \quad t>a .
$$


A combination of Lemma 1.1 (iv) and (i) now shows that

$$
\lambda_{1}\left\{\int_{a}^{t} Q(s) d s\right\} \leqslant \lambda_{1}[-V(t)]+\lambda_{1}[V(a)]
$$

for $t>a$. Passing to the limit in (3.10) as $t \rightarrow \infty$ we obtain

$$
\lim _{t \rightarrow \infty} \lambda_{1}[-V(t)]=\infty \text {. }
$$

We now apply (1.7) with $A=-V(t)$ and use (3.11) to find that

$$
\lim _{t \rightarrow \infty} \lambda_{1}\left[V^{2}(t)\right]=\infty \text {. }
$$

Finally an application of (1.8) using (3.12) yields

$$
\lim _{t \rightarrow \infty} \operatorname{tr}\left[V^{2}(t)\right]=\infty \text {. }
$$

This implies

$$
\lim _{t \rightarrow \infty} t^{-1} \operatorname{tr}\left\{\int_{t_{0}}^{t} V^{2}(s) d s\right\}=\infty
$$

i.e.,

$$
\lim _{t \rightarrow \infty} t^{-1} \lambda_{1}\left\{\int_{t_{0}}^{t} V^{2}(s) d s\right\}=\infty
$$

Applying now the trace functional to both sides of (3.8) and rearranging terms we get

$$
\operatorname{tr}\left[V(t)+\int_{t_{1}}^{t} V^{2}(s) d s\right]=\operatorname{tr}\left[V\left(t_{1}\right)\right]-\operatorname{tr}\left\{\int_{t_{1}}^{t} Q(s) d s\right\}
$$

after the modification in the lower limit of integration.

Use of the assumption (2.8) in (3.16) shows that the latter is bounded above by a term of the form $M t$ for some $M$. Lemma 2.1 now applies and so

$$
\liminf _{t \rightarrow \infty} t^{-1} \lambda_{1}\left\{\int_{t_{1}}^{t} V^{2}(s) d s\right\}<\infty
$$

which contradicts (3.15). The proof is now complete.

The author gratefully acknowledges, with thanks, the assistance of the referee who noted that the original proof of Theorem 2.1, subject to a slight modification and use of the weaker hypothesis (2.8), gave rise to the same conclusion. Originally (2.8) was stated as (2.9) with " $\lambda_{n}$ " replaced by "tr".

\section{REFERENCES}

1. R. Bellman, Introduction to matrix analysis, 2nd ed., McGraw-Hill, New York, 1970.

2. W. A. Coppell, Disconjugacy, Lecture Notes in Math., vol. 220, Springer-Verlag, New York, 1971.

3. G. J. Etgen and J. F. Pawlowski, Oscillation criteria for second order self-adjoint differential systems, Pacific J. Math. 66 (1976), 99-110.

4. P. Hartman, Ordinary differential equations, Hartman, Baltimore, Md., 1973.

5. Oscillation criteria for self-adjoint second order differential systems and "principal sectional curvatures", J. Differential Equations 34 (1979), 326-338.

6. D. Hinton and R. T. Lewis, Oscillation theory for generalized second order differential equations, Rocky Mountain J. Math. 10 (1980), 751-756.

7. W. T. Reid, Ordinary differential equations, Wiley, New York, 1971.

Department of Mathematics, University of Ottawa, Ottawa, Ontario, Canada, K1N 9B4 